Portland State University

PDXScholar

$10-1-2008$

\title{
Biomimetic Subwavelength Antireflective Gratings on GaAs
}

Chih-Hung Sun

University of Florida

Brian J. Ho

University of Florida

Bin Jiang

Portland State University

Peng Jiang

University of Florida

Follow this and additional works at: https://pdxscholar.library.pdx.edu/mth_fac

Part of the Mathematics Commons

Let us know how access to this document benefits you.

Citation Details

Sun, Chih-Hung, Ho, Brian, Jiang, Bin, Jiang, Peng (2008) Biomimetic Subwavelength Antireflective Gratings on GaAs. Optics Letters, 33, 2224-2226

This Article is brought to you for free and open access. It has been accepted for inclusion in Mathematics and Statistics Faculty Publications and Presentations by an authorized administrator of PDXScholar. Please contact us if we can make this document more accessible: pdxscholar@pdx.edu. 


\title{
Biomimetic subwavelength antireflective gratings on GaAs
}

\author{
Chih-Hung Sun, ${ }^{1}$ Brian J. Ho, ${ }^{1}$ Bin Jiang, ${ }^{2}$ and Peng Jiang ${ }^{1, *}$ \\ ${ }^{1}$ Department of Chemical Engineering, University of Florida, Gainesville, Florida 32611, USA \\ ${ }^{2}$ Department of Mathematics and Statistics, Portland State University, Portland, Oregon 97201, USA \\ *Corresponding author: pjiang@che.ufl.edu
}

Received July 29, 2008; accepted August 15, 2008;

posted August 29, 2008 (Doc. ID 99567); published September 29, 2008

\begin{abstract}
We have developed a simple and scalable bottom-up approach for fabricating moth-eye antireflective coatings on GaAs substrates. Monolayer, non-close-packed silica colloidal crystals are created on crystalline GaAs wafers by a spin-coating-based single-layer reduction technique. These colloidal monolayers can be used as etching masks during a $\mathrm{BCl}_{3}$ dry-etch process to generate subwavelength-structured antireflective gratings directly on GaAs substrates. The gratings exhibit excellent broadband antireflective properties, and the specular reflection matches with the theoretical prediction using a rigorous coupled-wave analysis model. These bioinspired antireflection coatings have important technological applications ranging from efficient solar cells to IR detectors. (C) 2008 Optical Society of America

OCIS codes: $050.2770,220.4241,310.1210,310.6628$.
\end{abstract}

Gallium arsenide (GaAs) is a technologically important semiconductor that has been widely used in optoelectronics, such as vertical cavity surface-emitting lasers [1], near-IR photodetectors [2], and highly efficient concentrator solar cells [3-5]. However, owing to the high refractive index (RI) of GaAs $\left(n_{\mathrm{GaAs}}>3.6\right.$ for visible wavelengths), more than $30 \%$ of incident light is reflected back from the substrate surface. This greatly reduces the efficiency of GaAs-based optoelectronic devices. To suppress the unwanted reflective losses, vacuum-deposited multilayer dielectric (e.g., $\mathrm{MgF}_{2} / \mathrm{ZnS}$ ) antireflection coatings (ARCs) have been developed [6,7]. Unfortunately, these multilayer ARCs are expensive to fabricate owing to the stringent requirement of high vacuum, material selection, and layer thickness control. Additionally, thermal-mismatch-induced lamination and material diffusion of the multilayer ARCs limit the device performance at high power densities [3].

Inspired by the broadband antireflection of microstructured corneas of moths, which consist of nonclose-packed arrays of sub-300 $\mathrm{nm}$ nipples [8], subwavelength antireflective structures have been extensively exploited [9-17]. These structures can reduce reflection over a wider range of wavelengths and exhibit much improved thermal stability than conventional multilayer ARCs. However, scalable production of subwavelength ARCs is not a trivial task for the current top-down nanolithography technologies (e.g., electron-beam lithography and interference lithography) [11,13]. Bottom-up colloidal lithography, which uses self-assembled colloidal crystals as deposition or etching masks to pattern periodic nanostructures $[18,19]$, provides a much simpler and inexpensive alternative to nanolithography in creating subwavelength gratings $[16,20]$. Unfortunately, traditional colloidal assemblies suffer from low throughput, small areas, incompatibility with standard microfabrication, and limited close-packed crystal structures.

We have recently developed an inexpensive and scalable colloidal lithography technology for creating moth-eye antireflective gratings by using monolayer, non-close-packed silica colloidal crystals prepared by a simple spin-coating technique as etching masks [21,22]. However, significant obstacles have been encountered when we employ this nonlithographic technique to GaAs substrates. Only disordered colloidal monolayers can be assembled on GaAs by spin coating. This greatly reduces the uniformity and reproducibility of the antireflective performance of templated ARCs. Here we report a generalized singlelayer reduction (SLD) technique that enables reproducible production of monolayer colloidal crystals on a variety of substrates that otherwise lead to the formation of disordered crystals by the traditional spin-coating approach $[23,24]$. We also demonstrate the scalable production of broadband ARCs on crystalline GaAs substrates, and their antireflective properties are characterized by both experimental measurements and theoretical simulation.

Figure 1(a) shows a typical scanning electron microscope (SEM) image of a colloidal monolayer con-

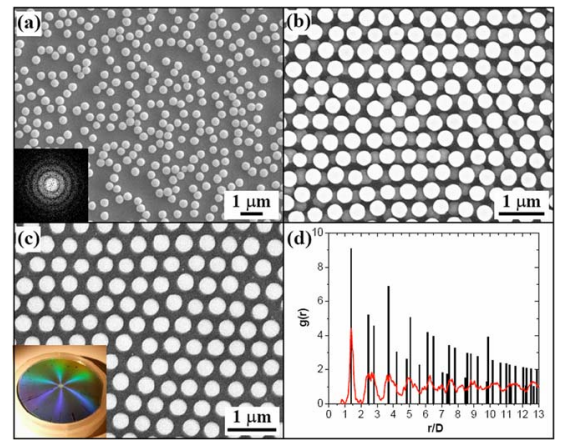

Fig. 1. (Color online) (a) Disordered colloidal monolayer formed on GaAs by traditional spin coating. Inset, Fourier transform of the image. (b) Double-layer colloidal crystals formed on GaAs. (c) Monolayer colloidal crystal formed by SLD. Inset, photograph of a monolayer crystal formed on a 2 in. GaAs wafer illuminated with white light. (d) Pair correlation function calculated from a low-magnification SEM image. For comparison, the PCF for an ideal hexagonal lattice with $\sqrt{2} D$ inter particle distance is also shown (black lines). 
sisting of $320 \mathrm{~nm}$ silica spheres prepared on a crystalline GaAs substrate [N-type, (100), University Wafer] by the standard spin-coating process [23,24]. The particles are completely disordered as confirmed by the rings in the Fourier transform of the SEM image. Contrary to the direct formation of colloidal monolayers by spin coating, where the substrate plays a crucial role in determining the resulting crystalline quality $[23,24]$, double-layer colloidal crystals are firstly assembled in SLD. Figure 1(b) shows a double-layer colloidal crystal prepared by spincoating colloidal silica suspensions $(20 \mathrm{vol} \%$ in nonvolatile ethoxylated trimethylolpropane triacrylate monomer) at $8000 \mathrm{rpm}$ for $90 \mathrm{~s}$. The non-closepacked hexagonal arrangement for both the top- and bottom-layer particles is clearly evident. After removing the top-layer particles by sweeping using a cleanroom Q-tip under flowing water, crystalline colloidal monolayers with non-close-packed structure are formed as shown in Fig. 1(c). The distinctive six-arm Bragg diffraction star formed on the $2 \mathrm{in} .(5.08 \mathrm{~cm})$ GaAs wafer [inset of Fig. 1(c)] indicates the hexagonal ordering is over the whole wafer surface $[24,25]$. The interparticle distance of the non-close-packed colloidal crystal is determined to be $1.40 D$, where $D$ is the diameter of silica spheres, by the first peak of the pair-correlation function [PCF, Fig. 1(d)], $g(r)$, which is calculated as $g(r)=1 /\langle\rho\rangle d n(r, r+d r) / d a(r, r$ $+d r)$, where $d n$ counts the number of spheres that lie within a spherical shell, $d r$, of radius $r$ from an arbitrary origin; $d a=2 \pi r d r$ for a particular radial distance $r$; and $\langle\rho\rangle$ is the average particle number density. Figure 1(d) also shows the positions of the oscillating PCF peaks agree well with those obtained from a perfect hexagonal lattice with $\sqrt{2} D$ interparticle distance. Preliminary results show the SLD technology can be easily and reproducibly applied to other important substrates, such as $\mathrm{Ni}, \mathrm{Cr}$, glass, and poly(methyl methacrylate). Only disordered monolayers are formed on these substrates by the traditional spin-coating technique [24].

The periodically arranged silica particles can then be used as etching masks during a $\mathrm{BCl}_{3}$ reactive ion etch (RIE) process operating at 7.5 mTorr chamber pressure, 20 SCCM (SCCM denotes cubic centimeters per minute at standard temperature and pressure) $\mathrm{BCl}_{3}, \mathrm{RIE}=100 \mathrm{~W}$, and inductively coupled plasma (ICP) $=300 \mathrm{~W}$ on a Unaxis Shuttlelock reactive ion etcher to generate arrays of cone shaped nanopillars. Figures 2(a) and 2(b) show the templated GaAs structures after 9.5 min RIE and removing templating silica particles by a $2 \%$ hydrofluoric acid wash. Interestingly, we observe the formation of mushroom-like microstructures. The "stems" of the mushrooms are cone-shaped and are caused by the isotropic etching of GaAs by reactive chlorine ions. The polymer wetting layer between the templating silica spheres and the substrate [26], which protects the GaAs surface immediately under the particles from being etched, causes the formation of the flat "caps" of the mushrooms. This unusual microstructure has been used to develop superoleophobic sur-

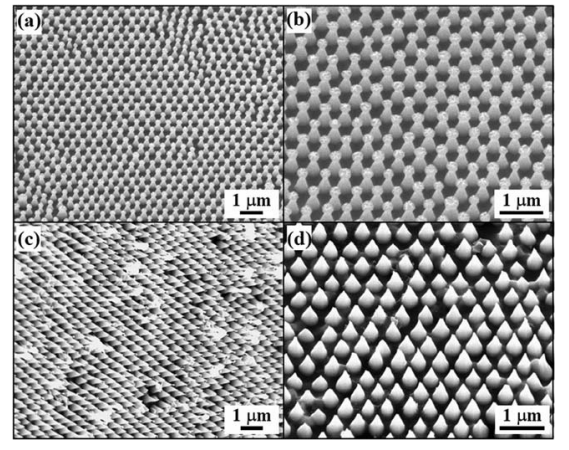

Fig. 2. (a) Templated mushroom-like GaAs microstructures after 9.5 min $\mathrm{BCl}_{3}$ RIE. (b) Higher magnification image of (a). (c) Conical nipples formed after $12 \mathrm{~min} \mathrm{BCl}_{3}$ RIE. (d) Same sample as (c) after a brief ultrasonication in acetone.

face [27], though it is not easily available by conventional top-down and bottom-up techniques. Longer dry etching leads to sharper conical stems and smaller caps as revealed by the SEM image of a 12 min etched sample in Fig. 2(c). The residual caps can finally be removed by a brief ultrasonication in acetone, resulting in the formation of conical GaAs gratings as shown in Fig. 2(d). The preservation of the long-range hexagonal ordering and the interparticle distance of the original silica colloidal monolayer throughout the templating process are clearly evident by comparing the SEM images of Figs. 1 and 2 .

The specular reflectivity of the templated subwavelength gratings are evaluated using visible- and near-IR reflectivity measurement at normal incidence by using an Ocean Optics HR4000 spectrometer. The solid curves in Fig. 3 show the measured reflection from a bare GaAs wafer and the nanocone grating as shown in Fig. 2(d). The wafer exhibits $>30 \%$ reflectivity, while much reduced reflection $(<3 \%)$ is obtained for the templated subwavelength grating. Importantly, the resulting moth-eye ARCs on GaAs are broadband, exhibiting consistent low reflection over both visible and near-IR wavelengths.

The optical measurement is complemented by theoretical simulation using a rigorous coupled-wave analysis (RCWA) model [28]. A conical profile is used to simulate the templated nipples as shown in Fig. 2(d). We divide the cone array into 100 horizontal circular layers. The intercone distance is defined as $\sqrt{2} D$ by the PCF calculation shown in Fig. 1(d). We can

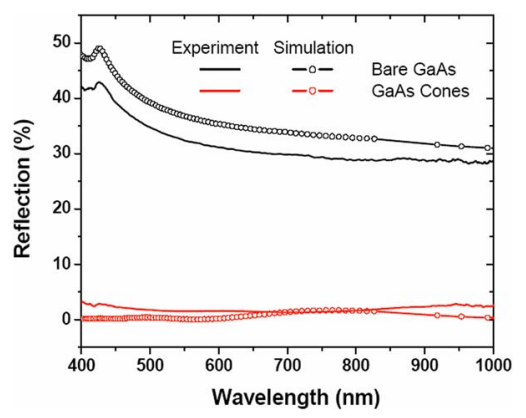

Fig. 3. (Color online) Experimental and RCWA-simulated specular reflection at normal incidence from a bare GaAs wafer and the conical grating as shown in Fig. 2(d). 
calculate the fraction of GaAs in each layer as $f\left(z^{*}\right)$ $=\pi\left(r^{*}\right)^{2} / \sqrt{3} D^{2}$, where $z^{*}$ and $r^{*}$ are the $z$ coordinate and the bottom radius of the cone, respectively. Based on the effective medium theory, the effective RI $n\left(z^{*}\right)$ of the layer at height $z^{*}$ can be approximated by $n\left(z^{*}\right)=\left[f\left(z^{*}\right) n_{\mathrm{GaAs}^{q}}+\left(1-f\left(z^{*}\right)\right) n_{\text {air }}{ }^{q}\right]^{1 / q}$, where $q=2 / 3$ $[8,29]$. We can then calculate the total reflectance of the layered system by solving Maxwell's equation to express the electromagnetic (EM) field in each layer and matching EM boundary conditions between neighboring layers. The complex RI of GaAs is used to calculate the resulting reflectance [30].

The curves with circles in Fig. 3 show the simulated reflection from a flat GaAs wafer and an array of conical nipples templated from $320 \mathrm{~nm}$ silica spheres. The calculated spectrum for the featureless substrate has the same shape as the experimental result. The simulated reflection from the subwavelength nanocone array also agrees reasonably well with the experimental spectrum. The excellent broadband antireflection properties of the templated conical gratings can be understood by mapping the calculated effective RI at $\lambda=700 \mathrm{~nm}$ across the height of $320 \mathrm{~nm}$ conical nipples (Fig. 4). For bare wafer, the RI changes sharply from air $(\mathrm{RI}=1.0)$ to bulk GaAs $(R I=3.772)$, while for templated nipples, the RI changes gradually from 1.0 to 3.41 and then to 3.772 . As the reflection $(R)$ from an interface between two materials with RI of $n_{1}$ and $n_{2}$ is governed by Fresnel's equation $R=\left(n_{1}-n_{2} / n_{1}+n_{2}\right)^{2}$ [29], the RI gradient exhibited by the conical nipples thus leads to very low reflection over a wide range of wavelengths.

In summary, we have developed a nonlithographic technology for creating moth-eye antireflection coatings on GaAs substrates. The technique is scalable and compatible with standard microfabrication, promising for developing more economic, efficient, and reliable GaAs-based optoelectronic devices.

This work was supported by the National Science Foundation (NSF) under grants CBET-0651780 and CBET-0744879, ACS Petroleum Research Fund, and the University of Florida Research Opportunity Incentive Seed Fund.

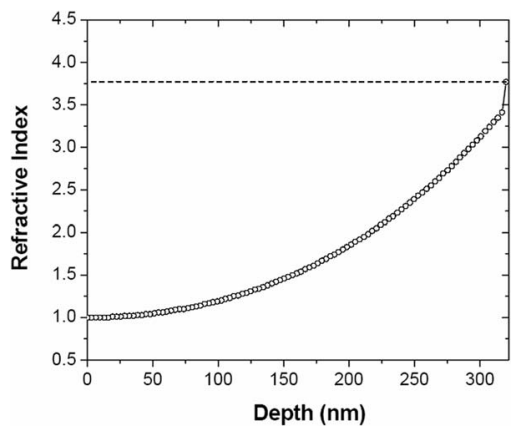

Fig. 4. Change of the calculated effective refractive index at $\lambda=700 \mathrm{~nm}$ from the wafer surface $($ depth $=0)$ to the bottom of templated GaAs nanocones with $320 \mathrm{~nm}$ height. The dotted line indicates the refractive index of bulk GaAs at the same wavelength.

\section{References}

1. J. K. O’Daniel, O. V. Smolski, and E. G. Johnson, IEEE Photon. Technol. Lett. 18, 445 (2006).

2. M. C. Wu, Y. H. Huang, and C. L. Ho, IEEE Electron Device Lett. 28, 797 (2007).

3. E. Oliva, F. Dimroth, and A. W. Bett, Prog. Photovoltaics 16, 289 (2008).

4. T. Yamada, A. Moto, Y. Iguchi, M. Takahashi, S. Tanaka, T. Tanabe, and S. Takagishi, Jpn. J. Appl. Phys. Part 1 44, L985 (2005).

5. J. Poortmans and V. Arkhipov, eds., Thin Films Solar Cells: Fabrication, Characterization and Applications (John Wiley \& Sons, 2006).

6. M. F. Schubert, F. W. Mont, S. Chhajed, D. J. Poxson, J. K. Kim, and E. F. Schubert, Opt. Express 16, 5290 (2008).

7. G. C. Farlow and L. A. Boatner, Opt. Mater. 8, 279 (1997).

8. D. G. Stavenga, S. Foletti, G. Palasantzas, and K. Arikawa, Proc. R. Soc. London Ser. B 273, 661 (2006).

9. J. R. Wendt, G. A. Vawter, R. E. Smith, and M. E. Warren, J. Vac. Sci. Technol. B 14, 4096 (1996).

10. P. B. Clapham and M. C. Hutley, Nature 244, 281 (1973).

11. A. Gombert, W. Glaubitt, K. Rose, J. Dreibholz, B. Blasi, A. Heinzel, D. Sporn, W. Doll, and V. Wittwer, Thin Solid Films 351, 73 (1999).

12. Y. Kanamori, K. Hane, H. Sai, and H. Yugami, Appl. Phys. Lett. 78, 142 (2001).

13. Y. Kanamori, M. Sasaki, and K. Hane, Opt. Lett. 24, 1422 (1999).

14. P. Lalanne and G. M. Morris, Nanotechnology 8, 53 (1997).

15. Z. N. Yu, H. Gao, W. Wu, H. X. Ge, and S. Y. Chou, J. Vac. Sci. Technol. B 21, 2874 (2003).

16. T. Lohmuller, M. Helgert, M. Sundermann, R. Brunner, and J. P. Spatz, Nano Lett. 8, 1429 (2008).

17. Y. F. Huang, S. Chattopadhyay, Y. J. Jen, C. Y. Peng, T. A. Liu, Y. K. Hsu, C. L. Pan, H. C. Lo, C. H. Hsu, Y. H. Chang, C. S. Lee, K. H. Chen, and L. C. Chen, Nat. Nanotechnol. 2, 770 (2007).

18. D. G. Choi, H. K. Yu, S. G. Jang, and S. M. Yang, J. Am. Chem. Soc. 126, 7019 (2004).

19. G. Zhang and D. Y. Wang, J. Am. Chem. Soc. 130, 5616 (2008).

20. B. G. Prevo, E. W. Hon, and O. D. Velev, J. Mater. Chem. 17, 791 (2007).

21. W. L. Min, A. P. Betancourt, P. Jiang, and B. Jiang, Appl. Phys. Lett. 92, 141109 (2008).

22. C. H. Sun, P. Jiang, and B. Jiang, Appl. Phys. Lett. 92, $061112(2008)$

23. P. Jiang and M. J. McFarland, J. Am. Chem. Soc. 126, 13778 (2004).

24. P. Jiang, T. Prasad, M. J. McFarland, and V. L. Colvin, Appl. Phys. Lett. 89, 011908 (2006).

25. P. Pieranski, Contemp. Phys. 24, 25 (1983).

26. C. H. Sun, W. L. Min, and P. Jiang, Chem. Commun. 2008, 3163 (2008).

27. A. Tuteja, W. Choi, M. L. Ma, J. M. Mabry, S. A. Mazzella, G. C. Rutledge, G. H. McKinley, and R. E. Cohen, Science 318, 1618 (2007).

28. M. G. Moharam, D. A. Pommet, E. B. Grann, and T. K. Gaylord, J. Opt. Soc. Am. A 12, 1077 (1995).

29. H. A. Macleod, Thin-Film Optical Filters, 3rd ed. (Institute of Physics Publishing, 2001).

30. E. D. Palik, ed., Handbook of Optical Constants of Solids (Academic, 1991) 